\title{
Development and transfer of interactive videodisc instructional technology
}

\author{
ANNE S. LEOPOLD and JOSEPH PSOTKA \\ U.S Army Research Institute for the Behavioral and Social Sciences, Alexandria, Virginia
}

\author{
Interactive videodisc instruction is described along with issues involved in the development \\ of the method.
}

Interactive videodisc technology is a relatively new medium for delivering instruction. Although some efforts by educators have been made to use this new technology, (e.g., Project BEST), it remains largely unused in schools. The U.S. Army is involved in several interactive videodisc instruction (IVI) projects. One of the major programs in this area involved the development of the Learning Strategies Curriculum for a population within the Army that receives adult remedial instruction. The Army Research Institute (ARI) is currently examining the potential for transfer of this instruction within the Department of Defense.

In addition, ARI is providing technical advisory service to the Berks County, Pennsylvania, Intermediate Unit School System for a pilot project to transfer this technology to the public education sector. The initial phase of this project is to adapt instruction from the Learning Strategies Curriculum to fit the needs and capabilities of high school students who are poor learners.

The first section of this paper provides a general description of IVI. This is followed by a review of development issues. The final section covers the transfer of this instructional technology to a new population.

\section{INTERACTIVE VIDEODISC INSTRUCTION}

Interactive videodisc instruction has many unique features that make it desirable for delivering instruction (Currier, 1983). Most IVI systems have the capability for stereo audio and real-time action. This latter feature is in sharp contrast to traditional computer-assisted instructional technology. A microcomputer disk holds enough storage space to store only $0.4 \mathrm{sec}$ of real-time video. In comparison, one videodisc can hold up to $30 \mathrm{~min}$ of real-time video on each side. The access time for a video-disc display is also dramatically shorter than that required for videotape. This allows the student more interaction per instructional hour using a random-access videodisc than using any other video medium.

The major components of an IVI system are the hard-

The authors' mailing address is: U.S. Army Research Institute for the Behavioral and Social Sciences, 5001 Eisenhower Avenue, Alexandria, VA 22333. ware, software, and courseware, as well as the videodiscs. Off-line materials may also be included to accomplish instructional techniques that are not cost effectively produced with the computer or videodisc. The hardware may consist of any of the traditional computer-based instructional components, such as a microcomputer, keyboard, black-and-white or color monitor, touch panel, light pen, and joy stick. The videodisc system adds on a videodisc player, and possibly a second monitor, thus allowing for the best of both video and computer-based instruction. The specific configuration of the peripherals depends on both the requirements of the instruction and the capabilities of the student population, in addition to the cost factor.

The software for computer-based videodisc instruction allows for conditional branching, scorekeeping, and data recording, along with the interaction between the computer and the videodisc player. Special software capabilities that provide unique features to the instructional system are available. Examples are the Spatial Data Management, Interactive Movie, and Vicarious Travel subsystems, which were developed by the Interactive Television Corporation (Levin, 1980). These programs were chosen for use with the Learning Strategies Curriculum. One of the major reasons for their inclusion was to increase the ease of system interaction for poor readers.

The Spatial Data Management subsystem allows the user to store and retrieve information using spatial locations and visual representations of the material. Related units of information may be clustered together on a data plane, giving visual cues to the relationships between units. The students may access units of instruction by using a joy stick to travel around the data plane and then "zooming in" to a figure or window. There may be several data planes for one lesson, each representing a more detailed level of information.

The interactive Movie subsystem allows students or an instructor to control the action and level of detail on the instruction. This software gives the student access to multiple groups of movie segments from the videodisc. The computer accesses various segments, as determined by the student responses and the desired instructional effect. The student may stop the action at any point in a film segment by using the freeze frame function of the software. 
The Vicarious Travel subsystem is accessed using a joy stick. This software gives the student control over the rate of speed and direction of travel. Through this feature, the student can take simulated trips on both a macro- (e.g., traveling over land) and a micro- (e.g., traveling through mechanical parts) level.

The courseware for IVI is basically in the form of traditional computer-assisted instructional material. This allows the student to interact with the computer using the question/answer method. When combined with the Interactive Movie subsystem, this prosaic and dull interaction is enlivened into a powerful and unique method of instruction. The system can simulate real-life situations, in which the student views and interacts with the world through the monitor and control panel. Learning is thus elevated to learning by doing. The student responds to scenarios at decision or choice points in the Interactive Movie subsystem program. Feedback is realistically immediate or delayed.

The final component of the system is the videodisc material. The videodiscs and courseware for the Learning Strategies Curriculum were developed by the Human Resources Research Organization (HumRRO). The material on a videodisc may be either static (still frames) or dynamic (movie segments). Generally, the more static the instruction is, the more computer instruction is in volved. This results in more instructional hours per videodisc. The major drawback in using available videodisc material is that it cannot be changed. Specifically, no new instruction may be added to the videodisc without incurring significant reproduction costs. (This capability, however, does exist in state-of-the-art technology.) There are, however, basic techniques for changing the insructional content of existing IVI material. A portion of a videodisc may be deleted from a lesson by programming around it, or the complexion of the instruction may be changed through additional computer programming or networking.

\section{ISSUES IN THE DEVELOPMENT OF IVI}

The development sequence for interactive videodiscs follows the basic methodology for developing any videotape or film, up to the point of computer networking. The analysis and design stages of production require a combination of skills on the part of the developer (the HumRRO Videodisc Group, 1982). In order to make full use of the motivational and in teractive capabilities of this medium, the developer should possess knowledge of the instructional techniques of both the film and the computer industries. This is necessary in order to visualize the final product's interplay among the movie segments, still frames, and computer programming. Once the final film is complete, the segments are arranged in the order they will appear on the videodisc. This order is driven by the planned computer networking of the instruction, in order to minimize unit access time.
Given this technical expertise, there remain two basic areas of concern for the developer of IVI: Choosing the right types of hardware and instructional design. There are many potential system configurations. The capabilities of the specific configuration chosen will impact on all aspects of the instructional development. The issue of what hardware to purchase should be decided after considering the instructional content, the anticipated student population, and the budget allocated for the project. Unfortunately, an emphasis may necessarily be placed on the cost of the individual hardware components, occasionally at the expense of their capabilities. Equally unfortunately, there is little research completed that will assist the developer in determining which configuration is best suited for teaching a specific content area to a given student population.

This problem is compounded by the changing state of the art in computer and videodisc technologies. New ad. vances are decreasing the cost and increasing the options for a potential user. Perhaps the best method to resolve the hardware issue is to let the instructional design dictate the purchase of system components.

The Army is currently engaged in several evaluation projects. The results are intended to assist the developer in choosing and implementing the appropriate instructional design. Several research questions are being addressed. The HumRRO Videodisc Group, for example, is examining the effects of learner control versus system control and hierarchical versus functional sequencing on student performance. Another research effort (Perkins, Salter, Perkins, \& Cook, in press) has investigated the effects of group size (individual, small group, and large group), mode of instruction (pedagogical vs. experiential), and group organization (leadership style) on student performance and preference. In addition, an inhouse research project is exploring the relationships between individual differences in attitudes toward testing and CAI, and student performance in the classroom and on the job.

Developing the appropriate design, however, is still an expensive, time-consuming effort. The average cost of developing, networking, and programming one videodisc instructional package is $\$ 60,000-\$ 100-000$. These costs are influenced by the amount and type of instructional design that are required. The greater the reliance on existing materials, such as training films, pictures, or selfpaced programs, the lower the development cost will be.

The promise of authoring aids and systems may help in lowering this estimate still further. Currently, however, the most viable method for introducing interactive videodisc instruction to the classroom is through transfer.

\section{TRANSFER OF IVI TECHNOLOGY}

Transfer of technology has been an important issue in applied psychology for over a decade. Traditionally, the 
transfer of technology has referred to moving the results of products of laboratory research, development, test, and evaluation (RDT\&E) into the field or classroom. Several approaches to achieving successful transfer of technology within the Army have been developed (Freda \& Shields, 1980; Seidel, 1975). These approaches center around the basic linear model for project implementation. The four steps of this model are: (1) requirements analysis, (2) RDT\&E, (3) information dissemination, and (4) institutionalization of products. This model, however, deals with the transfer of technology to the intended, or primary, user.

The transfer of instructional technology to an unanticipated, or secondary, user (as with transferring the Learning Strategies Curriculum to the public school system) takes on an abbreviated form. The requirements analysis is shortened, and the research and development of the instruction have already been completed. The new user may proceed with the second half of Step 2, at a great savings.

In fact, the average cost of transferring existing videodisc material to a new population is $\$ 200-\$ 20,000$ (not including the cost of hardware and evaluation). The lower range on this estimate indicates a direct transfer. This would be the case when the actual transfer consists of simply mailing a set of videodiscs and diskettes to a new user. The higher costs for transfer result if significant adaptations are required. An example of this situation would be the complete refilming of the videodiscs in order to provide symbolic modeling for another user population, or to insert aids for the hearing impaired.

Clearly, significant cost benefits may be derived from transferring existing IVI materials rather than developing new ones. The transfer of technology in general, is already in effect at the national and international level. The transfer of IVI materials and technology simply applies the existing processes of transfer as a viable means of disseminating this new instructional technology to the classroom.

\section{REFERENCES}

Currier, R. L. (1983, November). Interactive videodisc learning systems. High Technology, 3, 54-61.

Freda, J. S., \& Shields, J. L. (1980). An investigation of the adoption process in training technology transfer (Report No. 448). Alexandria, VA: U.S. Army Research Institute for the Behavioral and Social Sciences.

HumRRO VIDEOdisc Group. (1982). Instructional applications of spatial data management. Spatial Data Management, 2(3), 181-187.

Levin, S. (1980, March). Video disk-based spatial data management. Paper presented at the Office Automation Conference, Atlanta, GA.

Perkins, M. S., Salter, J. A., Perkins, M. N., \& Cook, W. A. (in press). Implementation of videodisc interpersonal skills training and assessment (VISTA). Alexandria, VA: U.S. Army Research Institute for the Behavioral and Social Sciences.

SEIDEL, R. (1975). A proposed integrated training system model (ITSM). In J. J. Rich and K. B. Van Pelt (Eds.), The future of the computer in Army training (Report No. CTS-TR-75-3, pp. 121-142. Ft. Monroe, VA: U.S. Army Training and Doctrine Command. 